\title{
Représenter les temporalités : outils et méthodes pour saisir les phénomènes de vacance commerciale
}

Representing the temporalities: tools and methods to identify the phenomena of commercial vacancy

\section{Sylvie Laroche}

\section{OpenEdition \\ Journals}

Édition électronique

URL : http://journals.openedition.org/developpementdurable/12153

DOI : 10.4000/developpementdurable.12153

ISSN : 1772-9971

Éditeur

Association DD\&T

Référence électronique

Sylvie Laroche, «Représenter les temporalités : outils et méthodes pour saisir les phénomènes de vacance commerciale », Développement durable et territoires [En ligne], Vol. 9, n² I Juin 2018, mis en ligne le 15 juin 2018, consulté le 03 mai 2019. URL : http://journals.openedition.org/ developpementdurable/12153; DOI : 10.4000/developpementdurable.12153

Ce document a été généré automatiquement le 3 mai 2019.

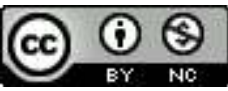

Développement Durable et Territoires est mis à disposition selon les termes de la licence Creative Commons Attribution - Pas d'Utilisation Commerciale 4.0 International. 


\title{
Représenter les temporalités : outils et méthodes pour saisir les phénomènes de vacance commerciale
}

\author{
Representing the temporalities: tools and methods to identify the phenomena of \\ commercial vacancy
}

Sylvie Laroche

\section{Vers une exploration des épaisseurs du territoire}

1 "On peut schématiquement figurer le milieu urbain comme une lentille adhérente au substrat terrestre, dont l'étendue et l'épaisseur mesurent la zone d'influence anthropique: plus l'agglomération est ancienne plus ses fondations sont profondes, plus elle est dense, plus sa canopée est élevée. Plus ses activités passées ont été industrieuses, plus elle s'est étendue dans ses faubourgs .» (Guillerme, 2010 : 39).

2 Dans le cadre d'une recherche expérimentale ${ }^{1}$, les temporalités urbaines furent investies en analysant les expériences quotidiennes et les temps longs de transformation du territoire. Pour mener cette exploration sur la sédimentation, la composition, voire la régénération des structures spatiales, nous nous sommes appuyés sur l'approche historiographique de l'environnement urbain défini par André Guillerme afin de croiser les données matérielles et immatérielles de la cité. Nous avons aussi associé la métaphore du territoire à la notion de palimpseste énoncée par André Corboz. Cette notion propose d'identifier l'organisation territoriale à travers son processus de stratification, qui produit avec une variété de vitesses et d'intensité une diversité de couches se superposant au fil du temps. Les éléments déterminant la transformation du territoire sont les phénomènes dynamiques de type géoclimatique, mais aussi - et c'est ce critère que nous allons prendre en considération dans notre recherche- les interventions 
humaines (Corboz, 1983). Ainsi, André Corboz invite à examiner les évolutions construites d'un territoire et les mutations de l'interaction entre les activités humaines et leur environnement naturel, et construit, afin de «les utiliser, comme des éléments, des points d'appui, des accents, des stimulants de notre propre planification " (Corboz, 2001 : 228). André Corboz poursuit cette réflexion sur la composition du territoire avec la métaphore de l'hyperville, où le territoire constitué d'une urbanisation généralisée est comparé à des lieux présentant certaines ressources disparates, mais reliées entre elles (Corboz, 2000). Dans la continuité de cette réflexion, "entre la gravité du palimpseste et la liquidité de l'hypertexte» (Marot, 2008), Sébastien Marot définit la notion de sub-urbanisme, qui propose aussi d'inverser nos investigations sur le territoire en privilégiant la composition du site à l'instar du programme. Quatre critères permettent de caractériser cette démarche : "La mémoire ou anamnèse des qualités du site, la vision du site et du projet comme processus plutôt que comme produits, la lecture en épaisseur, et non seulement en plan, des espaces ouverts, et enfin la pensée relative - une conception du site et du projet comme champ des relations plutôt que comme arrangements d'objets " (Marot, 2010 : 12). Il s'agit à travers ces quatre critères d'identifier les enjeux spatiaux, sociaux et temporels du territoire pour analyser la façon dont un projet se rapporte à son site. En s'appuyant principalement sur les postures énoncées par André Corboz et Sébastien Marot, Noha Gamal Saïd investit le temps long de la ville à travers l'écologie sensible et les phénomènes d'ambiance architecturale et urbaine. Cette approche permet d'identifier la temporalité de l'expérience quotidienne tout en mettant en valeur la dimension diachronique dans laquelle l'ambiance évolue (Gamal Saïd, 2014).

3 La prise en compte de la multiplicité des temporalités urbaines nous permet ainsi de saisir la transformation ordinaire des lieux (Brayer, 2013), et plus spécifiquement d'appréhender la mutation des commerces et leurs influences au sein d'un environnement construit.

\section{Singularités des espaces vacants}

\subsection{Définitions de la vacance commerciale}

Dans cette exploration des temporalités urbaines, nous nous focaliserons sur les représentations de la stratification du territoire et sur la portée opératoire des modes d'investigation établis. Pour ce faire, nous prendrons appui sur les situations particulières des espaces vacants dans la ville, et plus précisément des espaces vacants de la fonction commerciale. Cette investigation porte principalement sur les commerces de détail sédentaires, mais elle inclut aussi les services marchands tels les banques, qui ont un impact spatial, que ce soit par les flux, l'animation ou le paysage généré.

La singularité de la vacance commerciale est identifiable dans la diversité des définitions proposées pour ce statut. Dans le rapport intitulé «La revitalisation commerciale des centres-villes", rédigé par l'Inspection générale des finances et le conseil général de l'Environnement et du Développement durable, «la vacance commerciale évalue l'ajustement de l'offre de locaux d'un territoire à la demande " (Duhamel, 2016 : 14). Cette définition ne représente pas l'ensemble des situations de vacances commerciales. Pascal Madry (Madry, 2009 : 257) apporte des précisions en distinguant :

- «la vacance conjoncturelle: local inexploité pendant une courte période (en raison par exemple de travaux ou de changement de propriétaire); 
- la vacance structurelle : local inexploité pendant une longue période (en raison par exemple d'une perte durable de commercialité)».

6 Ces deux distinctions permettent de préciser les enjeux du mode de calcul du taux de vacance commerciale, à savoir le rapport en pourcentage entre le nombre de locaux commerciaux vacants et le nombre total de locaux commerciaux présents dans un ensemble commercial. Par convention, un taux de vacance inférieur à $7 \%$ traduit un phénomène de vacance conjoncturelle; un taux de vacance supérieur à $7 \%$ traduit un phénomène de vacance structurelle ${ }^{2}$.

\subsection{Historique de ce phénomène}

7 Pourtant, ce phénomène de vacance commerciale n'est pas récent. Il est déjà présent au milieu des années 1980 au Royaume-Uni : «Plusieurs collectivités locales se saisissent alors du phénomène comme symptôme d'une crise d'attraction de leur territoire, liée à un affaiblissement de leur base économique, et tentent d'y remédier à travers les premières politiques de redynamisation commerciale, ciblées sur leurs centres-villes (town center management en 1986) » (Bouvier et Madry, 2017: 7). En 2003, un recensement de l'activité commerciale en Amérique a révélé un destin inquiétant concernant de nombreux malls : près de quatre mille malls sont fermés, au moins un mall sur cinq est voué à la fermeture, quatre cents malls sont abandonnés par la seule enseigne Wall-Mart (Violeau, 2003). L'apparition des friches commerciales devient importante; une expression désigne ces bâtiments abandonnés : les dead malls. En Belgique, les cellules vacantes se sont multipliées depuis la fin de la Seconde Guerre mondiale, provoquant des situations de crise sociale et urbaine (Grimmeau et Wayens, 2016).

8 En France, le mode de conception des équipements commerciaux semble arriver à une rupture par rapport à la démarche antérieure, développée durant les Trente Glorieuses (Moati, 2011). Depuis 2008, nous assistons à une remise en cause du modèle de planification du commerce (INSEE, 2009). En 2011, le député Daniel Fasquelle a sondé l'ampleur de ce phénomène de vacances commerciales et a proposé des pistes législatives pour remédier à ce problème (Fasquelle, 2011). Depuis 2013, Pascal Madry nous interpelle sur l'évolution des vacances commerciales au sein de Procos, la fédération pour l'urbanisme et le développement du commerce spécialisé. Dans les différentes études éditées par cette fédération, le taux de locaux inoccupés ne cesse d'évoluer, tant dans les centres-villes que dans les centres commerciaux de périphérie $(7,2 \%$ en $2012 ;-7,8 \%$ en $2013 ; 8,5 \%$ en 2014 et $9,5 \%$ en 2015) (Madry, 2011). On s'aperçoit que cette vacance commerciale est un phénomène durable et qu'il tend à s'intensifier. Ce processus croise des enjeux préalablement définis pour l'analyse des friches urbaines. En effet, en prenant en compte les singularités des temps propres à la vacance, c'est-à-dire des espaces aux statuts transitoires et provisoires, le processus de composition des espaces vacants peut faire écho aux temporalités des friches urbaines identifiées par Charles Ambrosino et Lauren Andres (2008), à savoir : «Le temps de veille » où les friches constituent un espace d'expérimentation pour des acteurs issus de la société civile; « Le temps de la légitimité et construction de "coalitions opportunes" avec la visibilité des acteurs informels et la suggestion de nouvelles manières de produire la ville; «Le temps de la projection: la friche ressource foncière et idéelle » où se cristallisent les intérêts des acteurs privés, publics, et des habitants de la friche. L'évolution des friches urbaines ou des commerces vacants 
croise ainsi des imaginaires et des conflits de mutation d'un espace envisagé comme un laboratoire urbain ou un foncier disponible.

9 En parallèle à ces processus de renouvellement de la ville, les pratiques des usagers sont aussi façonnées par les temporalités spécifiques de la fonction commerciale dans la ville. Ces expériences quotidiennes croisent les rituels du dimanche (Lemarchand et al., 2016), les rythmes des pôles commerciaux (Soumagne, 2015) ou, comme le décrit Alain Metton (2001:168), "le temps du commerce n'est pas seulement le temps d'ouverture offert par les commerçants aux consommateurs pour réaliser les achats. C'est aussi, à travers la pratique commerciale des citadins-clients, l'un des meilleurs temps forts de la cité pour forger un mode d'urbanité».

\subsection{Prise en considération de la transformation des espaces vacants}

L'investigation du phénomène de vacance commerciale dans toute son ampleur et dans toutes ses temporalités, fait souvent évoquer des caractéristiques économiques, géographiques et démographiques comme facteurs de dévitalisation des centres-villes (Duhamel, 2016; Razemon, 2016). Dans le cadre de ce projet expérimental, nous avons donc choisi d'analyser les caractéristiques spatiales de ces espaces privés en déclin en cherchant :

- d'une part à identifier les éléments urbains et architecturaux favorisant cette désaffection;

- et d'autre part à saisir leurs perceptions au niveau des usagers.

11 Ainsi nous avons souhaité identifier ces caractéristiques en nous intéressant aux rez-dechaussée comprenant des locaux commerciaux, et plus exactement aux commerces vacants. L'hypothèse définie pour cette recherche est de considérer l'adaptabilité des équipements commerciaux comme un levier permettant de réinscrire ces espaces privés dans la ville. En effet, il s'agit de porter attention à l'aménagement de l'espace des commerces et d'envisager l'activité commerciale comme une "offre urbaine flexible" (Bourdin, 2010). Pour évaluer cette hypothèse, nous considérons la vacance commerciale comme des espaces observables, mesurables et repérables dans la ville; mais nous l'envisageons aussi comme un révélateur de dysfonctionnement entre la structure urbaine et la fonction commerciale. Cette double approche incite à prendre en compte les dimensions morphologiques de l'environnement autant que les dimensions physiques et sensibles des lieux marchands comme des critères pouvant favoriser la requalification, voire la reconversion de ce type d'espace.

\section{Représenter la vacance commerciale des faubourgs}

Le taux de la vacance commerciale pour la ville de Grenoble en 2015 est selon la fédération Procos de $6 \%$, et de 8,5\% selon la chambre de commerce et d'industrie. Nous avons voulu regarder dans une échelle plus fine comment se formalisait cette vacance. Nous avons choisi les quartiers péricentraux de la ville de Grenoble (quartiers Bajatière et Flaubert) situés entre le centre historique de la ville de Grenoble et les quartiers du Grand Sud, comprenant de nombreux projets d'aménagement et de rénovation urbaine. 
13 En raison de sa situation géographique, sa morphologie urbaine et sa composition programmatique, ce secteur est surnommé par les chargés d'urbanisme de la ville « le ventre mou grenoblois ». L'aspect chaotique pourrait qualifier ainsi ce territoire aux aménagements hétéroclites. Cependant, comme le démontre André Corboz (2009: 227), « la nébuleuse urbaine résulte d'une multitude de choix, qui sont tous rationnels, ou qui tendent à l'être, mais qui obéissent à des rationalités différentes, en concurrence les unes avec les autres ». Cette position peut être complétée par les réflexions de Michel Lussault, qui insiste sur la formation d'agencements complexes ne résultant pas "d'un quelconque mouvement spontané et naturel d'adaptation des hommes au milieu ni de leur soumission aveugle à des lois physiques ou à celles des structures sociales, pas plus qu'ils ne sont le résultat hasardeux des mouvements browniens d'individus utilitaristes. Ce sont des construits sociaux [...] des agencements formalisés de matières et d'idées, dotés d'attributs (l'échelle, la métrique, la substance, la configuration) par cette construction » (2007: 81).

Dans le cadre de ces quartiers grenoblois, le territoire était composé dans un premier temps d'un foncier agricole avec un habitat isolé. En 1920, la structure fut marquée avec l'implantation d'industries complétée entre 1945 à 1965 par un développement de l'habitat composé majoritairement de maisons. À la suite d'une désindustrialisation en 1965, le territoire fut densifié sans restructuration du maillage et d'un aménagement pour la voiture. Dans ce contexte, l'avenue Jean-Perrot traverse les quartiers péricentraux de Flaubert et Bajatière, et constitue une pénétrante à la métropole grenobloise d'une longueur de 4 kilomètres et d'une largeur constante de 25 mètres. Dans le cadre de cette recherche, nous nous focaliserons sur le tronçon situé dans la ville de Grenoble, long de 2 kilomètres. Il comprend actuellement 65 commerces et 13 locaux sont vacants.

\subsection{Protocole méthodologique pour explorer les temporalités urbaines}

Les objectifs de révéler les stratifications du territoire et d'identifier les expériences quotidiennes dans des quartiers possédant des singularités morphologiques, programmatiques et paysagères nécessitent d'adapter les représentations cartographiques mobilisées pour la géographie du commerce. C'est par la capacité de mise en relation de champs généralement séparés que l'outil du transect nous renseigne sur les multiples temporalités d'un lieu; qualités qui furent initialement exploitées dans les diagrammes élaborés par Patrick Geddes. La Valley section constituant une coupe réalisée en 1923 sur Edinburgh et sa vallée permet ainsi « d'interroger le type de relation qui s'établit entre les mots et les objets disposés dans des espaces proches, et en définissant un nouvel espace de relation qui contient tous les termes ou les objets présents dans le diagramme » (Viganò, 2012: 58). Nicolas Tixier s'appuie sur ce type de diagrammes et poursuit le développement de cet outil qui permet de mobiliser et d'articuler des connaissances en définissant le transect urbain comme "un dispositif se situant entre la coupe "clinique" et le parcours sensible empruntant à ces deux techniques pour les hybrider " (Tixier, 2012:251). La notion de temporalité se développe aussi dans la réalisation des transects, qui s'inscrit dans un processus de construction allant du recueil de données jusqu'aux opérations techniques nécessaires à leur réalisation (Palsky, 2017). 


\subsection{Réalisation des deux transects}

16 En prenant comme références ces expériences cartographiques, nous avons choisi de réaliser un transect de part et d'autre de l'avenue Jean-Perrot. Chaque représentation comporte :

- au centre : un extrait du plan cadastral comprenant le trait de coupe. Ce cadre central permet de représenter l'imbrication ou la superposition des réseaux des voies, les découpages fonciers et les constructions. Cette analyse urbaine classique met en évidence la structure originelle du territoire et ses modifications ;

- en partie supérieure : une élévation composée des plans des façades superposés à des photographies des bâtis. En s'appuyant sur la pratique photographique conceptuelle d'Edward Ruscha (1966), ces dessins techniques couplés aux clichés réalisés in situ permettent de se focaliser sur les détails des vitrines, de saisir les pratiques ou l'absence de pratiques dans l'espace public et identifier les différentes formes d'aménités proposées le long de cette infrastructure routière ;

- en partie inférieure : les plans et les photos des bâtis longeant l'avenue restitués dans l'espace et chronologiquement. Ces éléments iconographiques sont issus de deux relevés des commerces empruntés à l'organisme de l'Agence d'urbanisme de la région grenobloise et d'un diplôme réalisé à l'institut d'urbanisme de Grenoble, des permis de construire déposés entre 1950 et 2016 et accessibles aux archives de la ville de Grenoble. Cette approche fut complétée par les clichés réalisés par Google map, permettant de visualiser l'ensemble des campagnes «Street View » grâce à un curseur temporel. Cette investigation menée dans les archives, sur Internet, et éprouvée a comme objectif de saisir les disparitions, les mutations et les constructions des aménagements le long de cette avenue afin d'identifier les différentes strates visibles dans le présent. Pour restituer ces différentes temporalités, nous avons choisi de les exprimer par la décomposition spatiale d'un changement en une série d'images. Cette restitution s'appuie sur les modes de représentation des coupes temporelles élaborées par Noha Gamal Saïd (2014), c'est-àdire que les images sont situées en abscisse selon leurs localisations spatiales et en ordonnée selon leurs temporalités de formation. 
Figure 1 . Zoom sur une partie du transect du côté est de l'avenue Jean-Perrot
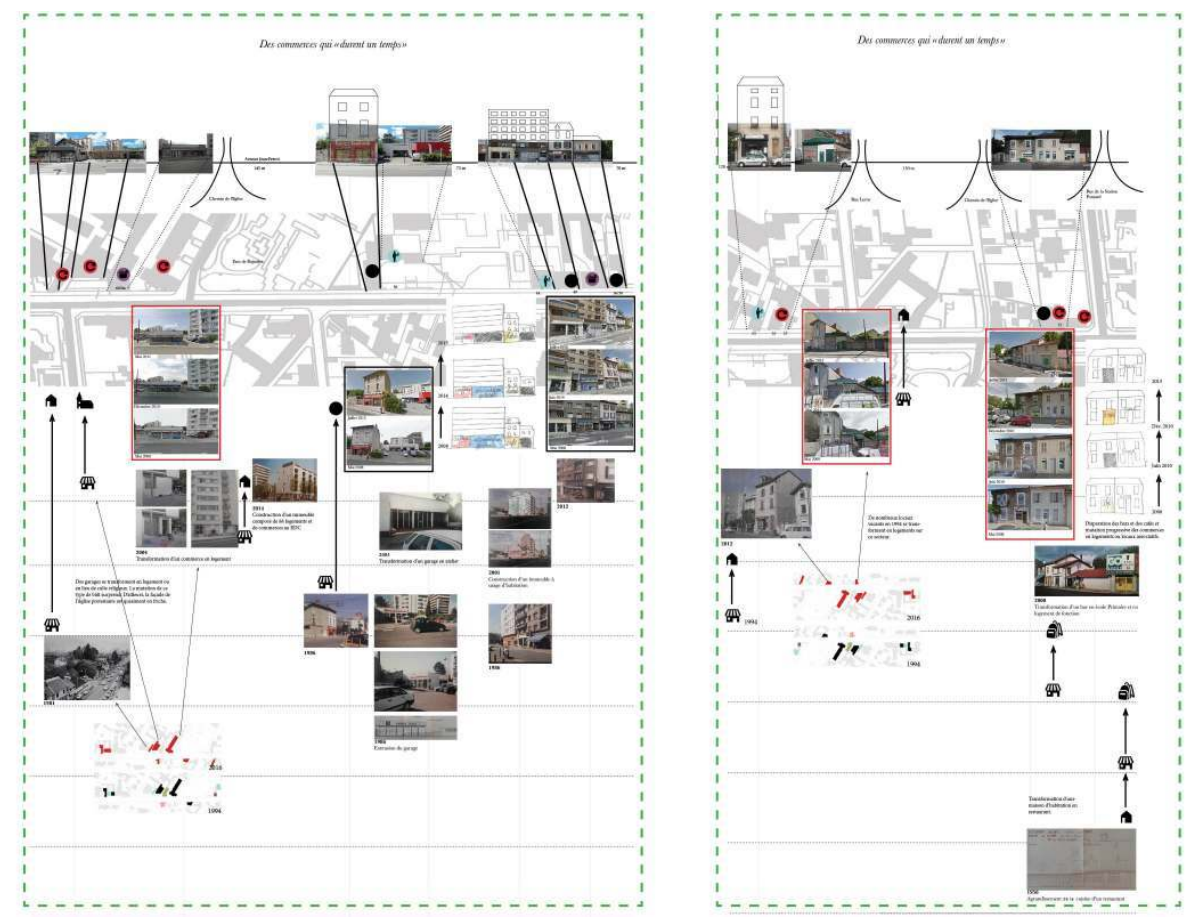

Source : Laroche et Lamothe, 2016

Cette investigation de l'avenue Jean-Perrot à travers la méthode des transects permet ainsi de se focaliser sur des espaces majoritairement traversés en voiture et pratiqués dans certains lieux par les piétons. Ensuite, le transect permet de croiser l'échelle du corps, du consommateur ou de l'usager à l'échelle de l'avenue traversant une métropole.

\subsection{Mises en débat des transects}

Les deux transects urbains ont permis de regrouper sans hiérarchiser les différentes données iconographiques, cartographiques et écrites pour caractériser cet aménagement linéaire. Il s'agit ensuite de pouvoir les communiquer, de partager et débattre entre les acteurs mobilisés par ces enjeux de la vacance commerciale. Nous avons adapté le dispositif de « la table longue » initié par Pascal Amphoux, qui propose « une façon plurielle de mettre le quotidien en débat. Une façon inédite de formaliser un imaginaire commun. Une façon pragmatique d'énoncer un cahier des charges ou les enjeux spécifiques d'un projet situé" (Amphoux, 2016: 41). Lors d'un séminaire organisé dans le laboratoire de recherche Ambiances Architectures Urbanités au sein de l'équipe Cresson ${ }^{3}$, nous avons élaboré un dispositif comprenant les deux transects collés de part et d'autre d'un large couloir. L'espace situé au centre de ces représentations cartographiques permettait aux experts de l'Agence d'urbanisme de la région grenobloise et aux membres des équipes scientifiques associées à cette recherche de débattre sur les données présentées. Cette scénographie obligeait les personnes à se déplacer en suivant le transect et à chercher des correspondances de chaque côté de l'avenue. Pendant le temps du séminaire, les experts étaient plongés en immersion dans les singularités du territoire choisi, ils ont pu ajouter des informations aux données présentées et mettre en débat les différentes représentations du territoire. 


\subsection{Restitution et partage des données} de données iconographiques, cartographiques et écrites collectées sur ce territoire. Nous avons développé une interface accessible sur Internet (https://sfr-uga.jeb-project.net), qui permet de placer sur un seul plan une vue aérienne, une vue "Street view » et de partager les données des commerces en activités, vacants et recyclés tout en les situant sur le territoire. Associée à chaque îlot comprenant des locaux commerciaux en rez-dechaussée, une fiche descriptive présente le nombre de cellules commerciales, le linéaire de vitrine entre les espaces privés et les espaces publics, la description spatiale et programmatique du local et les données iconographiques associées à cet espace. Cette interface permet de croiser une approche quantitative, mesurable, observable dans les aménagements passés et présents avec des approches qualitatives et incommensurables. Aussi fragmentaire soit-elle, il s'agit ainsi de constituer une mémoire urbaine des activités commerçantes passées et perceptibles encore actuellement.

Figure 2. Extrait d'une fiche descriptive (2016)

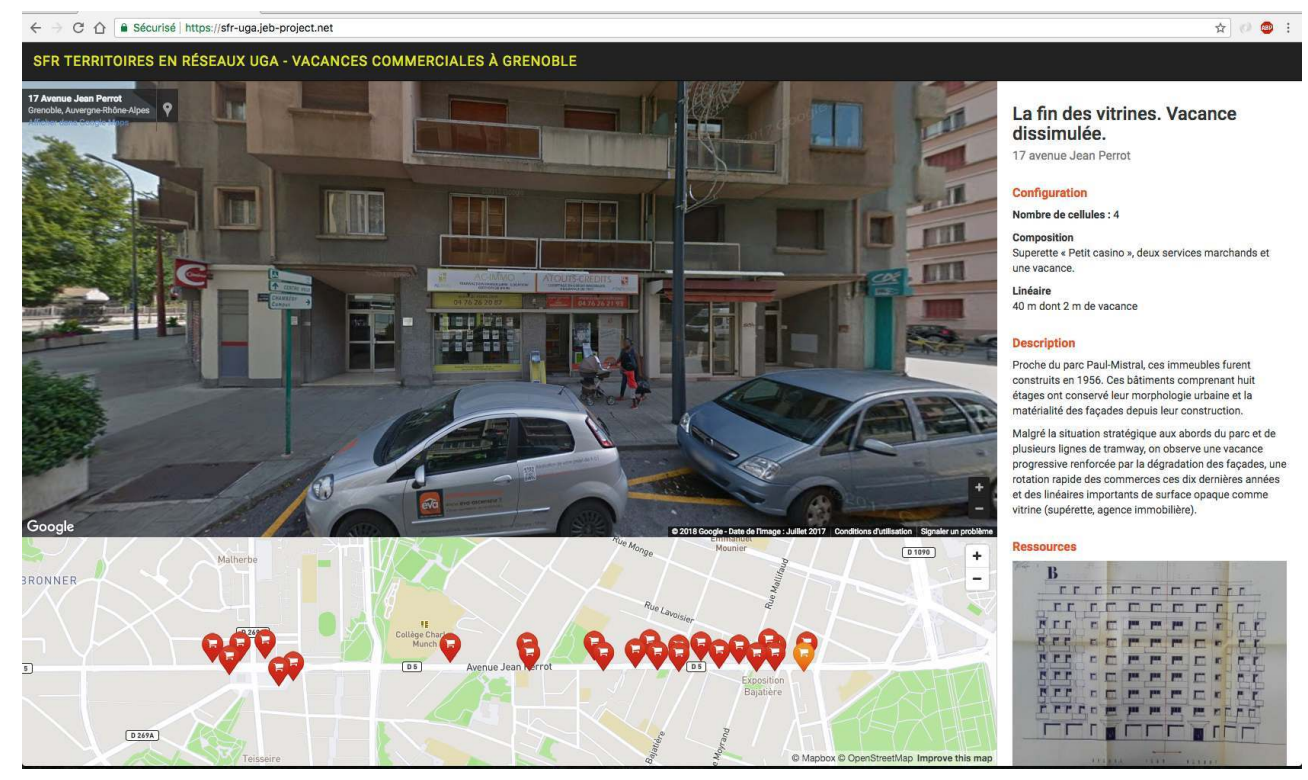

\section{Trois phénomènes de vacance commerciale}

Pour explorer les phénomènes de vacance commerciale, nous avons eu recours aux trois niveaux d'analyse définis par Grégoire Chelkoff (2001). Dans ses travaux, la forme correspond à l'articulation entre les dispositifs construits et l'environnement au niveau physique. Le formant repose sur l'expérience des phénomènes ressentis en action. La formalité relate le déploiement des pratiques en relation aux dispositifs et aux milieux sensibles. En s'appuyant sur ces notions, nous avons spécifié ces trois critères pour investir les multiples épaisseurs du territoire considéré, à savoir l'avenue Jean-Perrot :

- la forme est décrite à travers la dimension physique (matérialité), l'enveloppe des bâtiments qui comprend les plans verticaux (façades) et les porosités créées (ouverture, fenêtre). Ce registre vise à analyser les dispositifs sélectionnés d'un point de vue physique 
et morphologique. Ainsi, en nous appuyant sur les différents relevés accessibles dans les archives, sur Internet et éprouvés in situ, nous avons caractérisé l'architecture des rezde-chaussée longés ;

- les formants sensibles correspondent à l'expérience singulière des dispositifs architecturaux et des milieux mis à l'épreuve des déplacements des passants. L'enjeu de ce niveau d'analyse est d'appréhender l'expérience phénoménale des limites de commerces. Il s'agit plus précisément de décrire des configurations visuelles en observant la manière dont les commerces et les passants sont donnés à voir. La corrélation de ces mouvements vise à évaluer les qualités des aménagements le long de cette avenue ;

- les formalités caractérisent la manière dont les interfaces sont pratiquées, utilisées, détournées. Nous considérons que les dispositifs spatiaux et le contexte sensible mettent en jeu les modalités d'usages des lieux. Le critère de formalité permet ainsi de spécifier certains invariants dans les usages et les emplacements choisis par les personnes.

21 Les registres de la forme, du formant et de la formalité ne sont pas indépendants les uns des autres, au contraire ils apparaissent d'une manière concomitante. Ils s'articulent l'un à l'autre. La démarche est effectuée tout le long de l'avenue Jean-Perrot et de chaque côté de cette infrastructure. Cette approche nous a permis de saisir les caractéristiques des limites entre les rez-de-chaussée comprenant des locaux commerciaux et leurs contextes proches. À travers l'appréhension des milieux traversés visant à interroger leurs évolutions physiques, les qualités d'ambiances et l'adaptation potentielle des conduites, nous avons pu identifier trois phénomènes de vacances commerciales.

\subsection{La fin des vitrines}

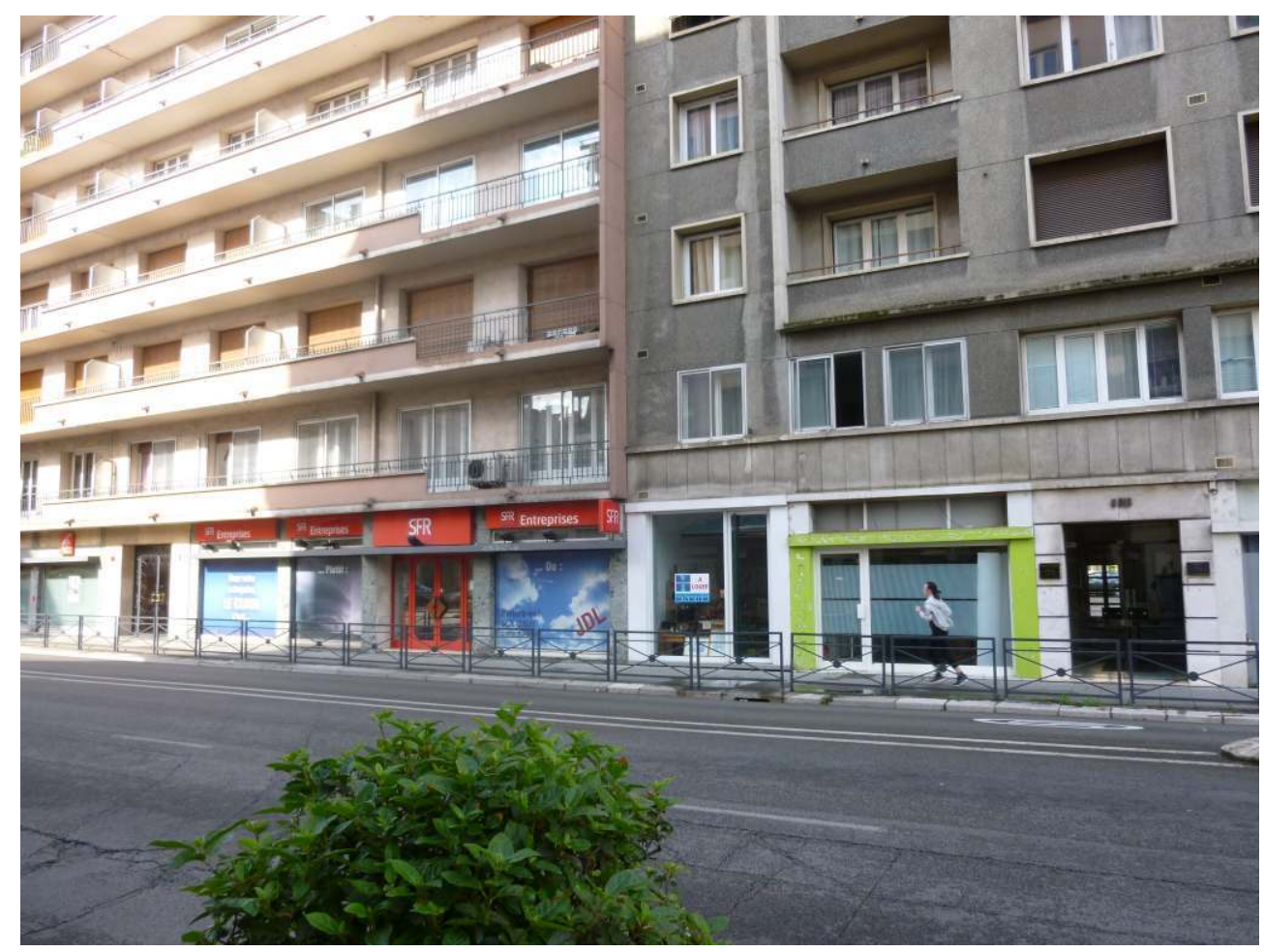

Source : Péron, 1993

Dans cette première séquence située au nord de l'avenue Jean-Perrot et au croisement avec le boulevard Clemenceau, la vacance commerciale est dissimulée au regard du 
nombre de locaux inoccupés (6 locaux vacants sur 25), mais de faible ampleur en mètre linéaire ( $8 \%$ de vitrines sont en situation de vacances).

Le premier critère pouvant expliquer ce phénomène de dissimulation des activités économiques peut être le manque de visibilité de trois commerces depuis l'avenue (immeuble en retrait d'un mètre ou séparé de l'avenue par un jardin large de huit mètres). En parallèle à ce critère morphologique, le deuxième motif de ce phénomène de vacance commerciale est le manque d'aménités dans les rez-de-chaussée. Cinq locaux (banques et services marchands) utilisent le procédé de vitrophanie, il s'agit d'un autocollant recouvrant l'ensemble de la surface de leurs vitrines. Pour celles-ci, les images sont encadrées par des bandeaux en partie supérieure des boutiques indiquant les principales informations de l'activité (horaire d'ouverture, spécificité de la vente, contact) et accompagnées d'une enseigne lumineuse reprenant le logo du commerce (un symbole vert pour une banque, le carré rouge pour la boutique de téléphonies mobiles). L'animation de ces commerces n'est pas visible depuis les espaces publics urbains, mais le regroupement des panneaux publicitaires et des enseignes aux couleurs vives forme un paysage distinctif le long de l'avenue. L'absence de visibilité à l'intérieur des locaux depuis le contexte environnant reprend les caractéristiques du hangar décoré énoncées dans L'enseignement de Las Vegas: "Quand les systèmes d'espace et de structure sont directement au service du programme et que l'ornementation est appliquée indépendamment d'eux, nous l'appellerons alors le hangar décoré. [...] Le hangar décoré est un abri conventionnel sur lequel des symboles sont appliqués » (Venturi et al., 1972: 97 et 100). La faible visibilité, voire l'absence d'activités commerçantes dans cette séquence transforme les rez-dechaussée en de simples "hangars" et les limites des infrastructures routières s'épaississent pour produire une animation uniquement dirigée vers les automobilistes.

Figure 3. Utilisation de la vitrophanie pour composer les vitrines

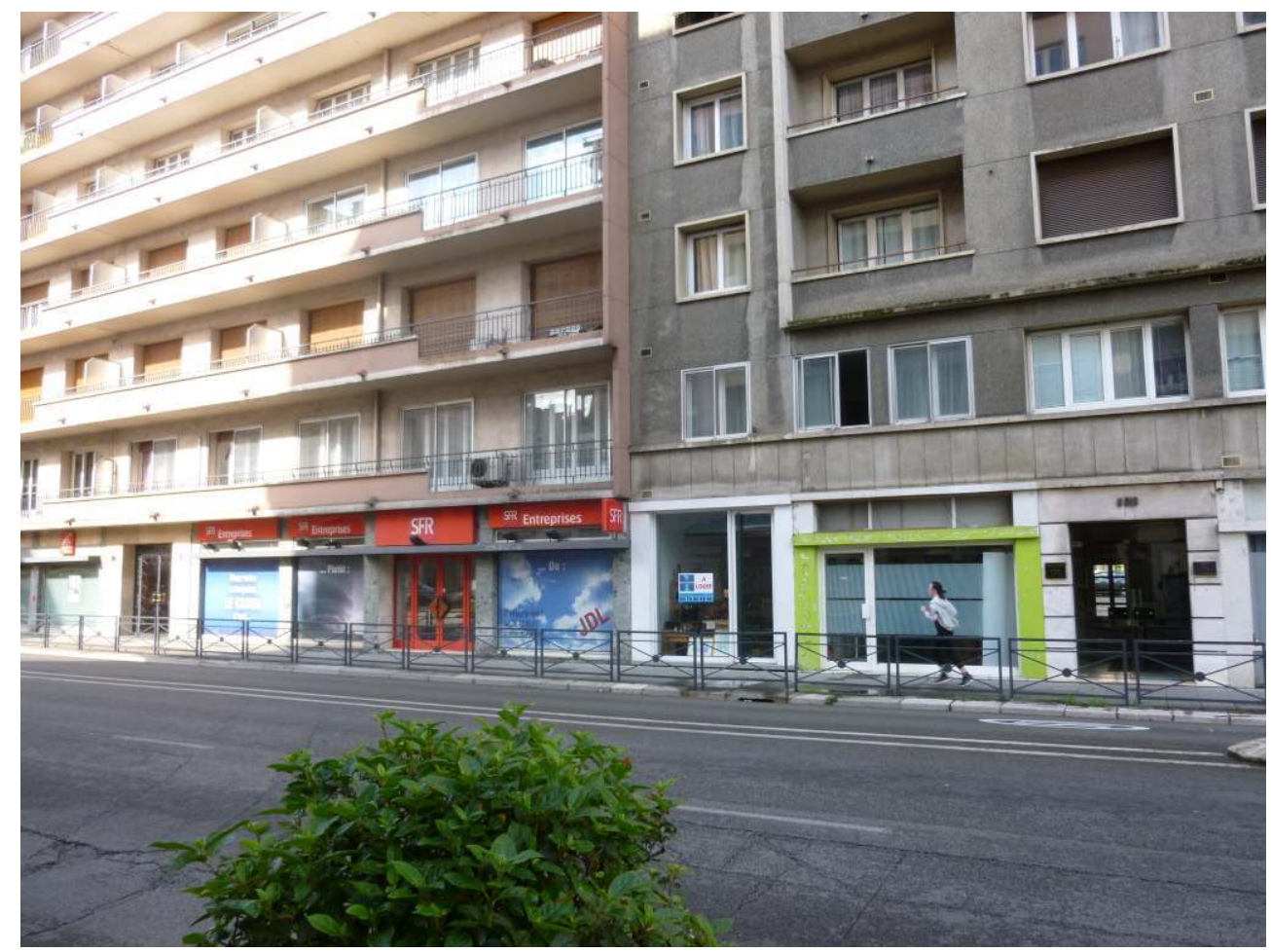

Source : Laroche, 2016 


\title{
4.2. Mitage commercial
}

\begin{abstract}
commerciale isolée. Seulement trois locaux sur douze sont vacants, cependant ces espaces sont fortement perceptibles depuis les espaces publics environnants. Les vitrines de ces locaux vacants sont parallèles à l'infrastructure routière et forment des surfaces importantes.
\end{abstract}

Figure 4. Local vacant, anciennement utilisé comme boulangerie

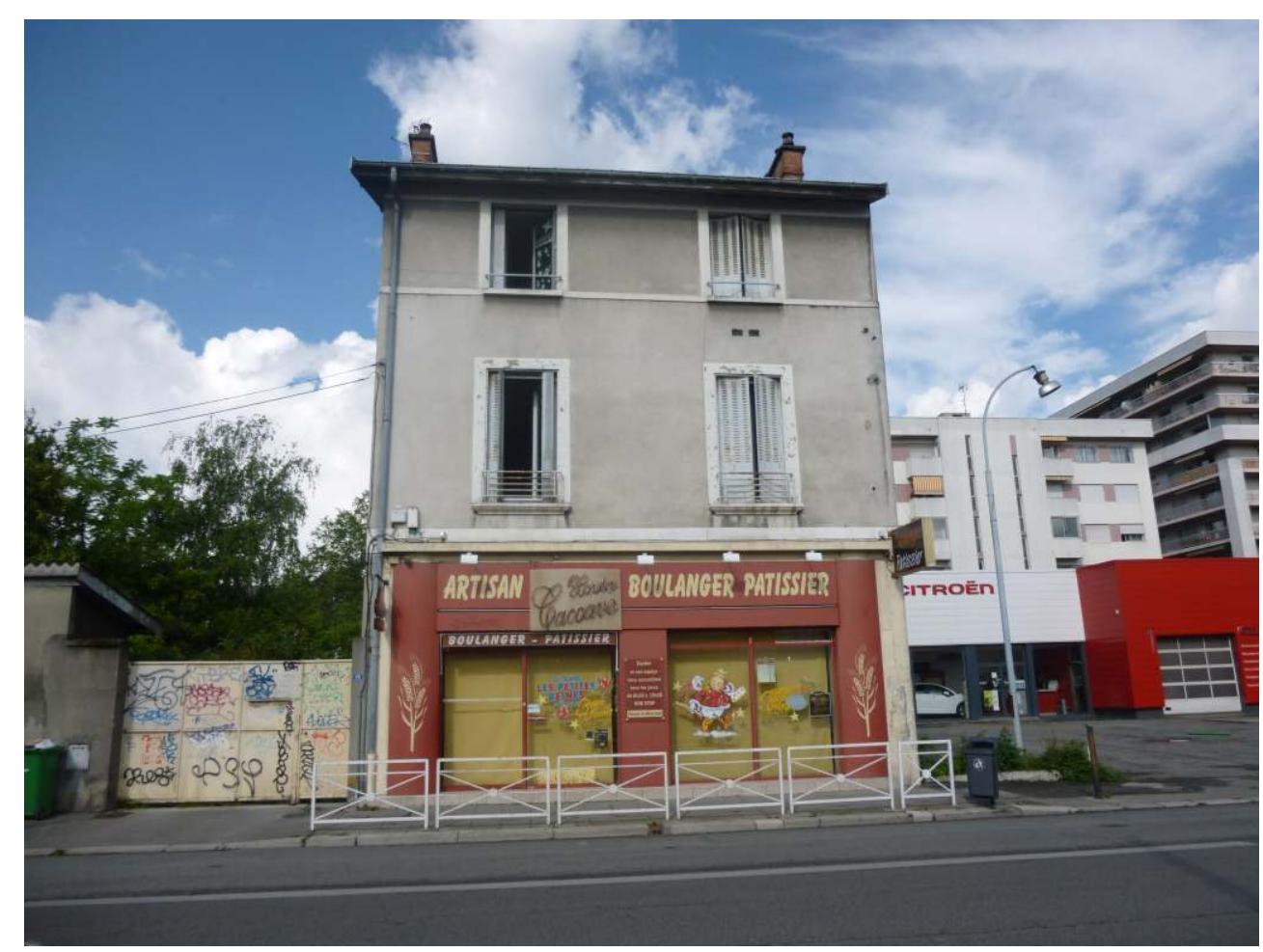

Source : Laroche, 2016

Ce mitage commercial est renforcé par la disparité des aménagements des rez-dechaussée des maisons concentrées au centre de l'avenue. Il se forme une forte reconversion programmatique des locaux initialement aménagés pour accueillir des bars, restaurants ou commerces de proximité transformés en logements, école privée, crèche associative, kinésithérapeute ou services marchands. Pour des raisons économiques et réglementaires, ces habitats, activités économiques ou associatives ont peu changé l'architecture des locaux commerciaux. Malgré l'aspect contraignant de ces bâtis construits vers 1950 (surface réduite, accessibilité complexe, faible ouverture), ces nouveaux programmes profitent d'une visibilité et d'un accès depuis une infrastructure passante au centre de la métropole, sont implantés dans des bâtis comprenant des cours fermées et préservent leur intimité grâce à l'utilisation de paravents, d'affiches, ou d'autocollants. Le recyclage des aménagements le long de cette avenue définit différents degrés d'hybridation entre les locaux commerciaux antérieurs et les programmes présents, qui révèlent comme un palimpseste les activités économiques passées invisibles en les ressortant dans l'environnement construit actuel.

Développement durable et territoires, Vol. 9, n² | Juin 2018 


\subsection{Vacances commerciales exposées}

Cette troisième séquence est marquée par une vacance commerciale de longue durée située uniquement au sud-est de l'avenue, dans le quartier Teisseire. Celui-ci fut construit dès 1950 et marqué par un projet de renouvellement urbain porté par l'architecte et urbaniste Philippe Panerai de 1997 à 2007. La majorité des habitants sont issus de classe modeste. Les commerces de ce quartier sont situés dans des bâtiments neufs et surélevés par rapport à la place publique dessinée par Philippe Panerai. Cet ensemble contient une dizaine de locaux appartenant majoritairement au bailleur social Actis et comprenant quatre cellules vacantes, dont deux restées vides depuis la livraison de cet aménagement.

Figure 5. Cellule commerciale vacante depuis 2007

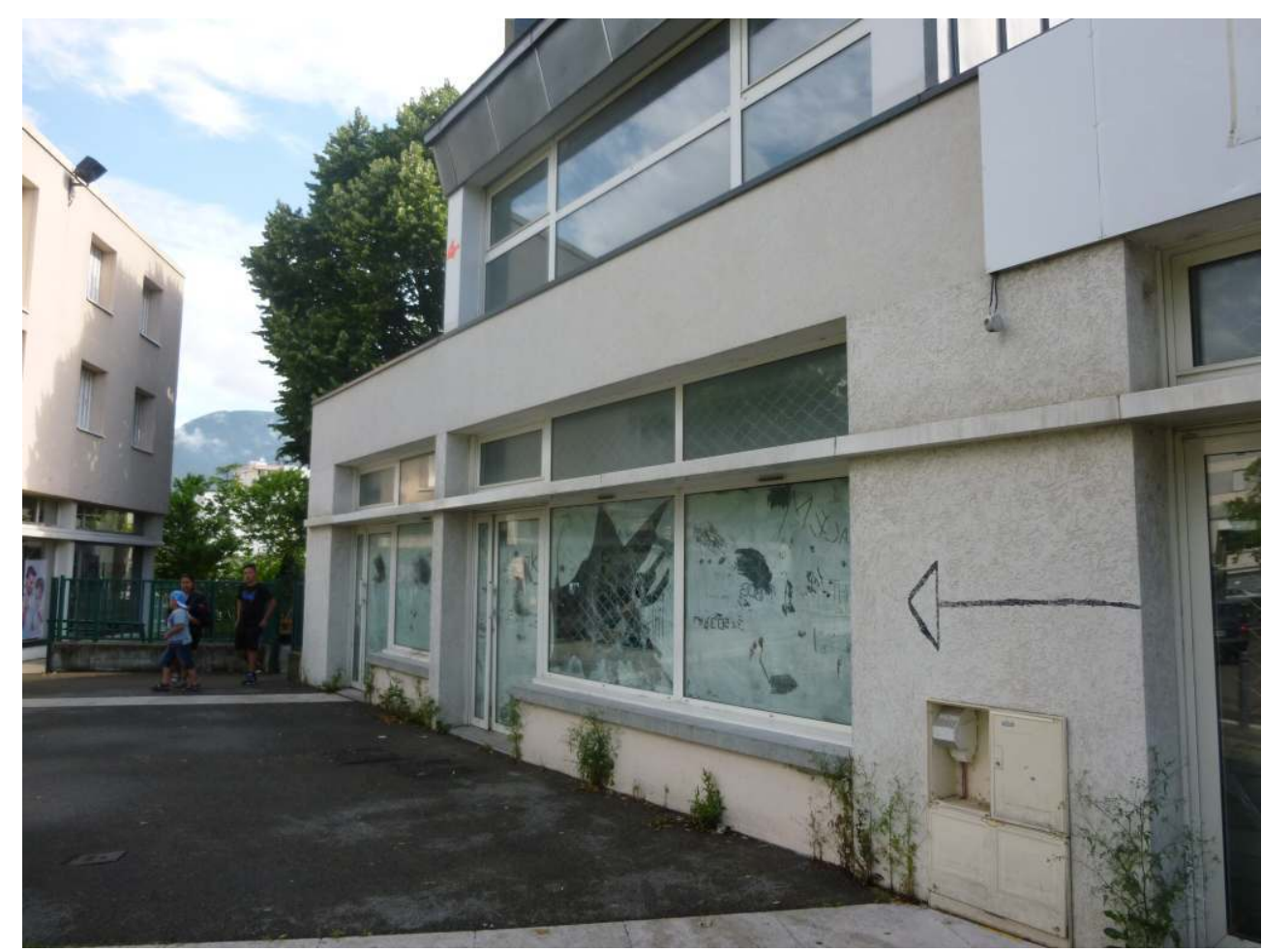

Source : Laroche, 2016

À l'inverse, à l'ouest de l'avenue, le quartier Malherbe est composé essentiellement de maisons individuelles et présente une population mixte à dominante de retraités propriétaires. Ce quartier comprend des commerces implantés depuis 20 ans dans des bâtis en limite de l'avenue, qui participent à la renommée de ce croisement: une boucherie chevaline, un magasin alimentaire «Biocoop» ou le tabac-presse. Malgré les aménagements récents du projet de renouvellement urbain et la qualité des espaces publics construits, le commerce prévu dans les rez-de-chaussée du quartier Teisseire ne permet pas d'amenuiser les confrontations entre ces deux quartiers, au contraire l'avenue demeure une fracture entre ces deux territoires et les cellules vides révèlent le dysfonctionnement entre ces structures urbaines. 


\section{Identifier les interfaces sensibles entre le privé et le public}

mises en débat, permettent d'analyser la composition historique et actuelle d'un territoire, il nous semble d'autre part que l'élaboration de ces protocoles méthodologiques peut participer à la définition du rapport entre l'espace privé et l'espace public.

Dans les trois séquences commerciales étudiées, nous n'avons pas observé une mutation de l'espace public sous la forme de privatisation (Sorkin, 1992; Davis, 1997), pas plus de phénomène d'aseptisation (Thomas, 2010) des aménagements en limite de l'avenue JeanPerrot. Nous avons davantage identifié des phénomènes de rétraction des activités présentes au niveau des rez-de-chaussée. En utilisant les vitrines comme écran pour certains services marchands comme les banques, ou en exposant des locaux vacants en direction des places publiques, ces rez-de-chaussée ne produisent pas d'animations en direction du contexte environnant. Il se forme, comme le définit Pascal Madry (2016: 240), « une relation complexe entre ville et commerce, qui peut se traduire par des évolutions dissociées de chacun de ses termes ». En parallèle de ce phénomène de rétraction, nous avons aussi identifié une forme de recyclage du bâti situé au centre de l'avenue avec l'inscription de programmes singuliers (école, crèche, artisan, etc.). Ce processus renvoie au phénomène de résilience défini comme une action permettant « de faire à un niveau local à travers la planification spatiale et la gouvernance pour minorer les conséquences écologiques de la société de consommation et les conséquences sociales de la "commercialisation" de la vie quotidienne » (Soumagne et al., $2013: 6$ ).

À travers ces deux phénomènes de rétraction et de résilience, il se dessine une opposition sur les objectifs de ces rez-de-chaussée offrant une neutralisation des espaces accessibles aux piétons, ou à l'inverse une aménité minime appropriable par certains usagers. Ainsi, on ne peut se contenter de désigner un espace selon son domaine privé ou public et la catégorisation des équipements commerciaux en fonction de leurs localisation, accessibilité et surface de vente. En effet, ces critères ne peuvent suffire pour qualifier l'influence d'un commerce vacant, actif ou recyclé sur les espaces et les urbanités avoisinants. En s'appuyant sur une approche fine et détaillée de l'environnement commercial, diverses nuances sensibles comme les ambiances sonores dans la cour d'école, la dynamique des personnes entrant et sortant d'un immeuble, ou encore le moirage d'une vitrine peuvent être repérés. Ces ambiances de basse intensité (Thibaud, 2014) révèlent les interactions entre l'espace et les usagers, entre le matériel et le subjectif, et identifient les différentes interfaces ordinaires entre le privé et le public pratiquées au quotidien.

\section{Les temporalités de conception des rez-de- chaussée dans la ville}

31 L'analyse des phénomènes de la vacance commerciale intéresse directement la réception par les usagers des vitrines situées dans les espaces privés en direction des espaces 
publics environnants, mais interroge aussi le processus de conception des rez-dechaussée dans la ville.

La conception de rez-de-chaussée actifs devient un enjeu majeur dans certains projets urbains de grande ampleur produisant des milieux urbanisés denses caractérisés par une composition urbaine plutôt compacte (Masboungi, 2013). Dans le cadre du projet urbain de la Chapelle Internationale, la démarche de l'agence AUC propose d'« intensifier les rezde-chaussée par des actions mineures » en concevant un double niveau permettant de mélanger des activités et des logements sur un volume (Poulin, 2013). L'architecte François Grether insiste sur la "flexibilité » des rez-de-chaussée pour pallier les attentes entre la livraison complète des bâtiments et la mise en place d'une animation dans ces nouveaux quartiers (Grether, 2013). Malgré ces expérimentations (GRAU Architectes, 2013), les images composées par les vitrines entre les espaces privés et publics reposent majoritairement sur la formation de parois dissimulant ou masquant les activités à l'intérieur des locaux. Ce mode de conception est critiqué selon leurs morphologies urbaines (Fromonot, 2012 ; Lucan, 2012), leurs programmations et les montages financiers (Mialet, 2011) ou leurs ambiances urbaines et architecturales (Laroche, 2014), et révèle les discontinuités du processus $d u$ projet avec des temporalités de programmation, conception, réalisation et utilisation asynchrones (Ramirez Cobo, 2017).

À l'opposé de ces modèles urbains, les quartiers péricentraux comprennent déjà une mixité urbaine et une diversité d'activités économiques. Ainsi, l'hypothèse méthodologique sur laquelle nous souhaiterions conclure s'appuie sur la pensée du projet comme producteur de connaissances défini par Paola Viganò (2012 : 172) :

34 "Des projets qui décrivent.

Tout acte de description est une tentative de reconstruction logique du monde et procède par des déconstructions, des effacements, des mises en relief. [...] La thèse est que les projets descriptifs et représentatifs sont des projets qui ne nient pas les caractéristiques structurelles de l'espace ni ne prétendent à les transformer, mais révèlent les éléments de rationalité de ces caractères mêmes. À partir de la constitution physique et matérielle du territoire, ils peuvent renvoyer à des pratiques et à des économies dotées elles aussi de différentes formes de rationalité ».

\section{Conclusion}

La déconstruction de l'espace et des temporalités urbaines permet d'une part d'analyser la composition d'un site à l'échelle du piéton comme celle du territoire, et d'autre part de dépasser l'opposition entre la désignation d'un espace selon son domaine privé ou public.

Ainsi, dans une démarche de projet urbain et architectural, nous pensons qu'il est bénéfique d'identifier la structure minimale de ces quartiers péricentraux en étant attentif aux différentes strates composant le territoire et aux expériences quotidiennes éprouvées dans les milieux investis. Cette attention peut s'appuyer sur les protocoles méthodologiques du transect pour croiser des données, les représenter puis débattre de cette investigation entre les acteurs du projet urbain et architectural.

Enfin, nous estimons que ces expérimentations menées in situ peuvent identifier le potentiel et les possibilités de ces territoires. En confrontant les aménagements existants et les projets de recyclages de l'urbain, nous pouvons échapper aux modèles génériques de planification du commerce remis en cause actuellement, et définir des propositions contextualisées. C'est en suivant cette double proposition, description et projection du 
territoire, que nous estimons que les outils et méthodes expérimentés peuvent participer au croisement des différentes temporalités urbaines.

\section{BIBLIOGRAPHIE}

Ambrosino C., Andres L., 2008 « Friches en ville : du temps de veille aux politiques de l'espace », Espaces et sociétés, vol. 3, n 134, p. 37-51.

Amphoux P., 2016, «Table longue », in Académie d'architecture avec la participation de la Cité de l'architecture et du patrimoine, Figurations de la Cité, catalogue exposition du 8 novembre-22 décembre 2016, p. 41-42.

Bourdin A., 2010, L'urbanisme d'après crise, Paris, Éditions de l'Aube.

Bouvier M., Madry P., 2017, «La vacance commerciale dans les centres-villes en France », Les Cahiers de l'Institut pour la ville et le commerce, vol. 1, p. 58.

Brayer L., 2013, « Appréhender, partager et concevoir le paysage en pratique à partir de dispositifs filmiques ", Articulo - Journal of Urban Research, n 4, URL : http:// articulo.revues.org/2241, consulté le 01/11/17.

Chelkoff G., 2001, « Formes, formants, formalités : catégories d'analyse de l'environnement urbain » in Grosjean M., Thibaud J.-P. (dir.), L'espace urbain en méthodes, Marseille, Parenthèses, p. 101-124.

Corboz A., 1983, « Le Territoire comme palimpseste », Diogène, n 121, p. 14-35.

Corboz A., 2000, « La Suisse comme hyperville », Le Visiteur, n 6, p. 112-129.

Corboz A., 2001, « Le Territoire comme palimpseste », in Corboz A. et Marot S., Le Territoire comme palimpseste et autres essais, Besançon, Éditions de l'imprimeur, collection « Tranches de villes », p. 209-229.

Corboz A., 2009, « Aptitudes territoriales, logiques concurrentes et implications politiques du projet d'urbanisme », in Corboz A., Tironi G., L'espace et le détour : entretiens et essais sur le territoire, la ville, la complexité et les doutes, Lausanne, L'Âge d'homme, p. 224-239.

Davis M., 1997, City of Quartz. Los Angeles, Capitale du futur, Paris, La Découverte.

Duhamel P.-M., Freppel C., Le Divenah J.-P., Munch J., Narring P., 2016, La revitalisation commerciale des centres-villes, l'Inspection générale des finances et le Conseil Général de l'Environnement et du Développement Durable, $471 \mathrm{p}$.

Fasquelle D., 2011, La vacance des locaux commerciaux et les moyens d'y remédier, rapport d'information (commission des affaires économiques), Assemblée Nationale, $n^{\circ} 3192$, déposé le 2 mars 2011, $54 \mathrm{p}$.

Fromonot F., 2012, «Production urbaine “à la française”. Que change la mondialisation ? ", Études foncières, $\mathrm{n}^{\circ}$ 157, p. 12-16. 
Gamal Saïd N., 2014, Vers une écologie sensible des rues du Caire : le palimpseste des ambiances d'une ville en transition, thèse de doctorat, urbanisme mention aménagement, École doctorale Sciences de l'homme, du politique et du territoire, École nationale supérieure d'architecture, , $464 \mathrm{p}$.

Gillio N. et Vial A., 2013, « Comment requalifier les espaces commerciaux ? », TechniCités, Éditions Certu, n²60, p. 20-22.

GRAU Architectes, 2013, Rez de ville - rez de vie, Paris, Pavillon de l'Arsenal.

Grether F., 2013, « Penser le projet à partir des rez-de-chaussée » in Masboungi A. (dir.), (Ré)aménager les rez-de-chaussée de la ville sous la direction, Paris, Le Moniteur, p. 44-50.

Grimmeau J.-P., Wayens B., 2016, « Les causes de la disparition des petits commerces (1945-2015) », Courrier hebdomadaire du CRISP, n 2301-2302, p. 5-114. URL : https:// www.cairn.info/revue-courrier-hebdomadaire-du-crisp-2016-16-page-5.htm, consulté le 01/11/17.

Guillerme A., 2010, « Historiographie de l'environnement urbain », in Coutard O., Lévy J.-P. (dir.), Écologie urbaines, Mayenne, Economica, p. 39-57.

INSEE, 2009, Le commerce en France, Paris, INSEE.

Marot S., 2010, L'art de la mémoire, le territoire et l'architecture, Paris, Éditions de La Villette, collection « Penser l'espace».

Laroche S., 2014, L'architecture commerciale à l'usage des villes : ambiances, pratiques et projets, thèse de doctorat, Architecture, École doctorale Sciences de l'Homme du Politique et du Territoire, École Nationale Supérieure d'Architecture de Grenoble, 346 p.

Lemarchand N., Mallet S., Paquot T. (dir.), 2016, En quête du dimanche, Gollion, Infolio, collection « Archigraphy Poche ».

Lucan J., 2012, Où va la ville aujourd'hui ? Formes urbaines et mixités, Paris, Éditions de La Villette.

Lussault M., 2007, L'homme spatial, la construction sociale de l'espace humain, Paris, Seuil.

Madry P., 2009, Dictionnaire. Pratique du commerce, Bruxelles, De Boeck Université.

Madry P., 2011, « Le commerce est entré dans sa bulle », Études foncières, n 151, p. 12-16.

Madry P., 2016, Ville et commerce à l'épreuve de la déterritorialisation, thèse de doctorat, urbanisme, université de Bretagne occidentale, $331 \mathrm{p}$.

Marot S., 2008, "Exile on main street, un manifeste relatif du sub-urbanisme », conférence « cycle Paysage », 19 novembre 2008, Pavillon de l'Arsenal.

Masboungi A., 2013, (Ré)aménager les rez-de-chaussée de la ville, Paris, Le Moniteur. Metton A., 2001, «Les temps du commerce sont-ils adaptés aux consommateurs? ", in Paquot T. (dir.), Le quotidien urbain : essai sur les temps des villes. La Découverte, Paris, p. 167-179.

Mialet F., 2011, Mixité fonctionnelle et flexibilité programmatique, Paris, Plan Urbanisme Construction Architecture, $43 \mathrm{p}$.

Moati P., 2011, La nouvelle révolution commerciale, Paris, Odile Jacob.

Péron R., 1993, La fin des vitrines. Des temples de la consommation aux usines à vendre, Cachan, École normale supérieure.

Palsky G., 2017, « Carte, temps et récit », in Besse J.-M., Tibergheim G., Opérations cartographiques, Arles, Acte Sud et ENSP, p. 56-69. 
Poulin C., 2013, « Intensifier les rez-de-chaussée par des actions mineures », in Masboungi A. (dir.), (Ré)aménager les rez-de-chaussée de la ville, Paris, Le Moniteur, p. 56-62.

Ramirez-Cobo I., 2017, L'incertitude comme levier de co-construction au prisme du projet urbain, thèse de doctorat, urbanisme mention aménagement, École doctorale Sciences de l'homme, du politique et du territoire, $464 \mathrm{p}$.

Razemon O., 2016, Comment la France a tué ses villes, Paris, Rue de l'échiquier, collection « Diagonales».

Ruscha E., 1966, Every Building on the Sunset Strip, Los Angeles, Édition Ruscha.

Soumagne J. (dir.) et al., 2013, Chronotope « Aménagement spatio-temporel pour des villes résilientes, ministère de l'Écologie, du Développement durable et de l'Énergie, 356 p.

Soumagne J. (dir.) et al., 2015, Temps et usages de la ville, Rennes, Presses universitaires, collection «Espaces et territoires».

Sorkin M. (dir.), 1992, Variations on a theme park. The new american city en the end of public space, New York, Hill and Wang.

Thomas R. (dir) et al., 2010, L'aseptisation des ambiances piétonnes au XXI e siècle, entre passivité et plasticité des corps en marche, Grenoble, Cresson, rapport de recherche $n^{\circ} 78,125 \mathrm{p}$.

Thibaud J.-P., 2014, « Ambiances de basse intensité », Colloque « Le quotidien filmé. Archives filmiques et Espaces publics », 3 novembre 2014, Cinémathèque de Grenoble.

Tixier N. (dir.), 2012, L'ambiance est dans l'air : la dimension atmosphérique des ambiances architecturales et urbaines dans les approches environnementalistes, Grenoble, Cresson, $250 \mathrm{p}$. Venturi R., Scott Brown D., Izenour S., 1972, L'enseignement de Las Vegas, Cambridge, MIT Press. Violeau J.-L., 2003, « Les friches du grand commerce », AMC, $\mathrm{n}^{\circ}$ 133, p. 22.

Viganò P., 2012, Les territoires de l'urbanisme. Le projet comme producteur de connaissance, Genève, MétisPresses.

\section{NOTES}

1. "Phénomènes de vacances et de friches commerciales. » Recherche 2016-2017 financée par la SFR « Territoires en réseaux », S. Laroche (UMR CNRS - MCC 1563 AAU) (cord.), C. Ambrosino, G. Novarina (UMR CNRS 5194 Pacte), C. Maumi (Les Métiers de l'Histoire de l'Architecture : édifices Villes et Territoires), R. Kazig (UMR CNRS - MCC 1563 AAU) et E. Lamothe (master design urbain). 2. Notons que ce seuil est aussi variable selon les définitions de la vacance commerciale : "Cette notion désigne l'état d'un local commercial non exploité pour une durée indéterminée. Au-delà de $6 \%$, la vacance commerciale d'une galerie ou d'un centre commercial devient structurelle et témoigne d'un déficit durable de commercialité » (Gillio et Vial, 2013).

3. Voir annonce et compte rendu de ce séminaire sur https://lcv.hypotheses.org/11166 consulté le $10 / 06 / 2017$. 


\section{RÉSUMÉS}

Cet article présente le protocole méthodologique expérimenté pour une recherche portant sur les temporalités des commerces et sur leurs influences au sein d'un environnement construit. L'investigation porte sur une infrastructure routière située dans la métropole grenobloise. L'objectif est d'analyser le processus de stratifications du territoire et d'identifier les expériences quotidiennes dans des quartiers possédant des singularités morphologiques, programmatiques et paysagères. Mais, il s'agit aussi de repérer des caractéristiques spatiales des commerces vacants qui singularisent ou homogénéisent la composition des rez-de-chaussée dans la ville.

This article presents the methodological protocol experimented for a research regarding the temporalities of the retail and their influences within a environment. The investigation is a road infrastructure in the Grenoble metropolitan area. We have chosen to study the morphological, programmatic and landscape qualitifies of the suburbs. In doing so, the goal is to analyze the process of stratifications of the territory and the experiences that one can have in these districts. It is also to identify the spatial characteristics of commercial vacancy which distinguish or homogenize the composition of ground floors in the city.

\section{INDEX}

Mots-clés : vacance commerciale, transect urbain, temporalités, expériences, stratification, faubourgs

Keywords : commercial vacancy, urban transect, temporalities, experiences, stratification, suburbs

\section{AUTEUR}

\section{SYLVIE LAROCHE}

Sylvie Laroche est docteure en architecture et chercheure au Cresson, UMR CNRS Ambiances Architectures Urbanités. Ses travaux portent sur la mutation des espaces commerçants dans les milieux urbains. ENSA Grenoble, sylvielaroche.archi@gmail.com 